\title{
Practical, Real-Time, and Robust Watermarking on the Spatial Domain for High-Definition Video Contents
}

\author{
Kyung-Su KIM $^{\dagger a)}$, Nonmember, Hae-Yeoun LEE ${ }^{\dagger \dagger}$, Member, Dong-Hyuck IM ${ }^{\dagger}$, \\ and Heung-Kyu LEE ${ }^{\dagger}$, Nonmembers
}

SUMMARY Commercial markets employ digital right management (DRM) systems to protect valuable high-definition (HD) quality videos. DRM system uses watermarking to provide copyright protection and ownership authentication of multimedia contents. We propose a real-time video watermarking scheme for HD video in the uncompressed domain. Especially, our approach is in aspect of practical perspectives to satisfy perceptual quality, real-time processing, and robustness requirements. We simplify and optimize human visual system mask for real-time performance and also apply dithering technique for invisibility. Extensive experiments are performed to prove that the proposed scheme satisfies the invisibility, real-time processing, and robustness requirements against video processing attacks. We concentrate upon video processing attacks that commonly occur in HD quality videos to display on portable devices. These attacks include not only scaling and low bit-rate encoding, but also malicious attacks such as format conversion and frame rate change.

key words: real-time video watermarking, robust video watermarking, practical video watermarking, high-definition videos

\section{Introduction}

In recent years, digital multimedia such as PDF, MP3, and HD-TV replace analog media such as books, tapes, and ana$\log$ TV signals. Since digital multimedia give an opportunity for end-users to create and distribute contents to other peoples, there are requirements to protect the contents of rights holders and trace illegal contents providers. Digital Rights Management (DRM) refers to the technology that supports legal distribution of digital contents [1], [2]. In DRM systems, digital watermarking is an important tool that protects the digital contents.

Figure 1 shows a basic structure of a DRM system. Rights holders and contents providers verify end-user's favorable content and initialize a packaging process. The verified content that requires protection is packaged into the protected contents by watermarking and encryption. After this process, the protected contents are available for distribution through physical distribution media or the Internet for online service. A wide variety of watermark applications require for watermarking algorithms to have low cost and low computational complexity, because end-users want to watch HD

Manuscript received July 23, 2007.

Manuscript revised November 19, 2007.

${ }^{\dagger}$ The authors are with the Department of Electrical Engineering and Computer Science, Korea Advanced Institute of Science and Technology (KAIST), Daejeon, Republic of Korea.

${ }^{\dagger \dagger}$ The author is with the School of Computer \& Software Engineering, Kumoh National Institute of Technology, Republic of Korea.

a)E-mail: kskim@mmc.kaist.ac.kr

DOI: 10.1093/ietisy/e91-d.5.1359

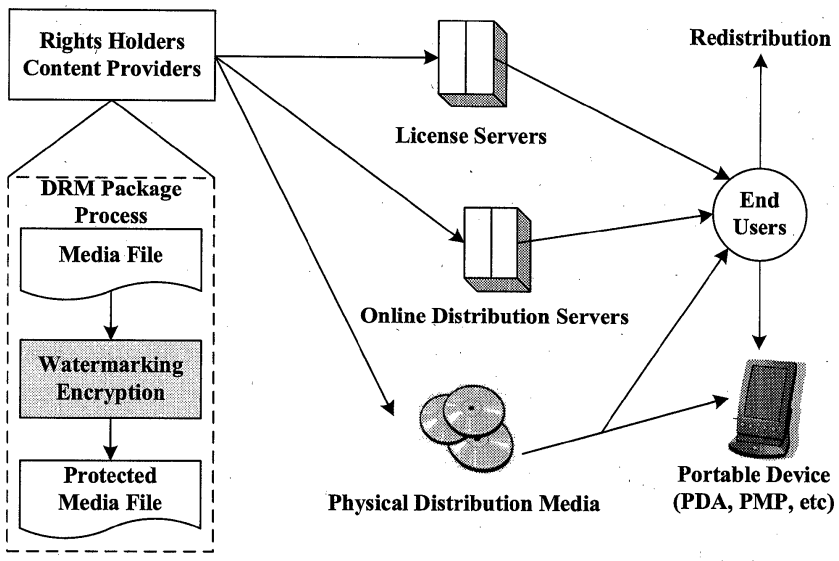

Fig. 1 Basic structure of a DRM system.

videos without delay or quality degradation through online distribution in DRM system. Real-time watermarking algorithms have applications such as broadcasting monitoring, owner identification, transaction tracking, content authentication, and so on. Real-time watermarking for high quality contents is not an exception any more [3].

\subsection{Practical Issues for Video Watermarking}

End-users who have been authenticated by license servers can access the protected contents freely. It means that endusers can download and record them into their storages without any restraints. In online movies and broadcasting marketplaces, their requests for high quality contents are rising rapidly and hence most video contents are made in digital formats and HD-TV quality (e.g., $1920 \times 1080$ or $1280 \times 720$ pixel resolution).

Since end-users would like to display the downloaded contents on their portable devices such as personal digital assistant (PDA), portable multimedia player (PMP), and $i \operatorname{Pod}^{\circledR}$, several video processing such as resizing, low bitrate encoding, transcoding, and frame rate change are inevitable. This is due to the fact that portable devices have limitation in the size of memory, the speed of processor, and the size of display screen. For example, 1920×1080 MPEG2 videos at 30 frame per seconds (fps) are converted into $320 \times 240$ MPEG-4 videos at 15 fps. Some distortions affecting viewing angles such as translation and rotation processing are beyond of our scope, because it does not commonly 
happen.

This paper is organized as follows. In Sect. 2, we review video watermarking research and analyze their disadvantages for practical applications. Sect. 3 explains our realtime and robust video watermarking against practical video processing and provides general guidelines for watermark embedding into HD videos. Experimental results are shown in Sects. 4 and 5 concludes.

\section{Background}

\subsection{Literature Review}

In the past few years, many papers have dealt with numerical attacks including compression, filtering, geometrical distortions, and bit-rate reduction in video watermarking. They embedded watermarks in compressed videos or in uncompressed raw videos.

Compressed domain video watermarking is faster than uncompressed domain video watermarking because it does not require fully decoded video streams and videos are stored in compressed formats. Langelaar and Lagendijk [4] proposed compressed domain video watermarking called differential energy watermark (DEW). Although the DEW has relatively low computational complexity and is robust against re-encoding of video streams, it is weak against transcoding when the structure of group of picture (GOP) is changed. Langelaar et al. [5] suggested watermark embedding by modifying codewords generated by a VLC. Since codewords are changed or remain unchanged according to watermark bits, the visual quality of watermarked videos can be guaranteed. However, it is hard to achieve good robustness to re-encoding and low bit-rate encoding, because it uses least significant bit (LSB) approaches. Another compressed domain video watermarking in VLC domain was proposed by Ling et al. [6]. It is called differential number watermarking (DNW), where watermark bits were embedded by using the number difference of tuples and threshold between sub-regions. DNW outperformed DEW in aspect of complexity, visual quality, capacity and robustness to transcoding. Wang and Pearmain [7] explained robust MPEG-2 video watermarking in DCT domain. They focused on typical geometric processing for bit-rate reduction, cropping, downscaling, and frame dropping. However, their algorithm can not directly adopt in different compressed formats such as MPEG-4 or H.264 and is vulnerable to transcoding.

Uncompressed domain video watermarking techniques have been suggested to deal the similar issues in compressed domain watermarking techniques. The most common method in uncompressed domain video watermarking is spatial domain video watermarking, where compressed video streams are decompressed into raw videos and then image watermarking techniques are applied to those raw videos. In general, watermarks are embedded by adding them to consecutive raw video frames and human visual system (HVS) should be preceded embedding process for visual quality and robustness [8]. The HVS is less sensitive to distortions around edges or texture areas. Delaigle et al. [9] designed spatial masking by combining an edge and texture discrimination to determine watermark strength. Xianghong et al. [10] suggested another HVS-based watermarking technique that was based on the modulation transfer function of HVS in discrete cosine transform domain, but such a transform is additional time-consuming work. Voloshynovskiy et al. [11] proposed a HVS function from noise visibility function (NVF) that used stochastic modeling of the cover image. The NVF function calculates local mean and local variance and then decides the strength of watermarks in pixel-by-pixel. Using the computation of NVF, watermarks are strongly embedded in textured and edge regions. Although HVS function from NVF exhibits a good visibility and robustness, calculating NVF takes high computational cost and is not appropriate for real-time applications. In [12], the watermark is embedded in blue channel due to the fact that the human eye is less sensitive to this channel. Some researches are focused on geometrical distortions [13], [14], where their schemes were usually based on converting a geometric invariant domain, template insertion, or using self-synchronizing watermark. However, it is also difficult to satisfy real-time embedding or extraction of watermarks and simultaneously make watermarks robust against practical video processing.

\subsection{Implementation Issues in Practical Perspective}

For real-time watermark embedding, several video watermarking schemes adapted to dedicated hardware such as a very long instruction word (VLIW) processor, a filed programmable gate array (FPGA), and an application specific integrated circuit (ASIC). In [15], real-time MPEG-2 video watermarking based on fractal coding was achieved by adapting it to both a 32 bit DSP embedded processor and a 32 bit VLIW multi-issue processor core. Since fractal coding based methods required the expensive computing time, they optimized code and ported it to the dedicated hardware. In [16], the customized ICs were employed to implement the watermark embedder and detector. All operations including filtering, FFT, and correlation operation were integrated inside one chip. Both methods have focused on visual quality rather than robustness and the size of videos was limited to VGA or QVGA. Although real-time embedding using hardware performs without platform constraint, it causes cost problem in installation and difficulty to maintain or upgrade hardware. Hence, real-time watermarking approaches on personal computer with a general purpose processor (GPP) are required to reduce the problems in cost and installation and upgrade for end-users.

Most compressed domain video watermarking use coefficients of DCT or DWT. It means that watermarking algorithms depend on transform itself and modification works are needed to embed into heterogeneous compressed video. For example, in order to apply DWT-based watermarking techniques on DCT-based compressed videos, the 
conversion of frequency transform domain should be required before watermarking a host video. However, video watermarking in spatial domain can be applied to DCTbased video coding as well as DWT-based video coding. Echizen et al. proposed a real-time video watermarking system which adapts to personal computer. They combined all processes including QVGA capture, watermark embedding, and MPEG-4 encoding and achieved real-time embedding by reusing the watermark strength of neighboring frames [17]. However, it only generated the MPEG-4 file and supported the QVGA format. They also described codec independent watermark embedding process which handled the VGA format directly [18]. Although real-time process was achieved by reusing watermark pattern generated by pre-process, their HVS function is computationally expensive because it needs to calculate local variances and also evaluates the flickering parameter for imperceptible embedding. Therefore, it is not suitable for HD size of videos due to computational complexity.

For real-time video watermarking for high resolution videos, spatial domain video watermarking methods mentioned in previous are not suitable and not practical. Likewise image watermarking techniques, spatial domain video watermarking requires HVS function to be robust and enhance visual quality. This paper addresses a practical, realtime, and robust video watermarking scheme for HD video in uncompressed domain, which directly adapt to an Intel Pentium $^{\circledR}$ platform personal computer. For real-time requirement, we simplify HVS function using separable linear filter and optimize it with Intel MMX technology. Also, we improve the perceptual quality of watermarked videos by applying a dithering technique. Using HD videos, we test robustness to practical video processing attacks and measure visual quality.

\section{Real-Time Video Watermarking}

We first explain watermark embedding and blind extraction of our video watermarking system. Practical issues as follows are then discussed; real-time processing to reduce computational cost, optimized HVS to enhance robustness, perceptual quality for invisibility, and false positive probability analysis.

\subsection{Structure of Watermarking System}

\subsubsection{Watermark Embedding}

Figure 2 illustrates the watermark embedding scheme. Let $X_{i}$ be a set of luminance component of host video frames and $Y_{i}$ be a set of watermarked video frames $(1 \leq i \leq N) . N$ is a total number of video frames.

The first step in embedding process is to generate basic patterns using private key and messages (e.g., copyright information, serial numbers, plain text, etc.) to be embedded. Basic patterns consist of $M 1-\mathrm{D}$ random patterns with a length $L$ that follow Gaussian distribution with zero mean

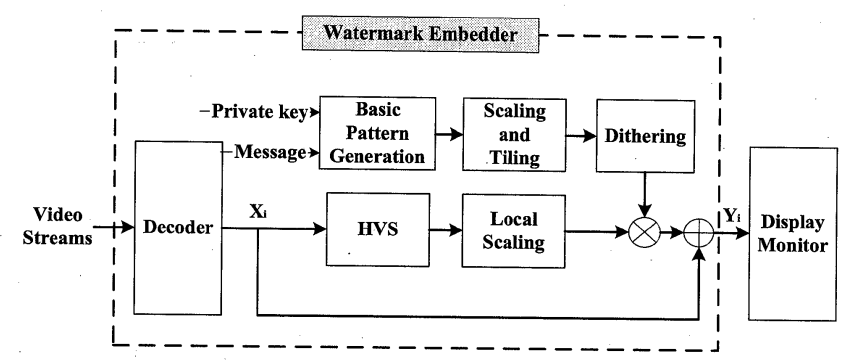

Fig. 2 Block diagram of real-time watermark embedding process: decoding video streams, embedding watermarks, and displaying on a screen.

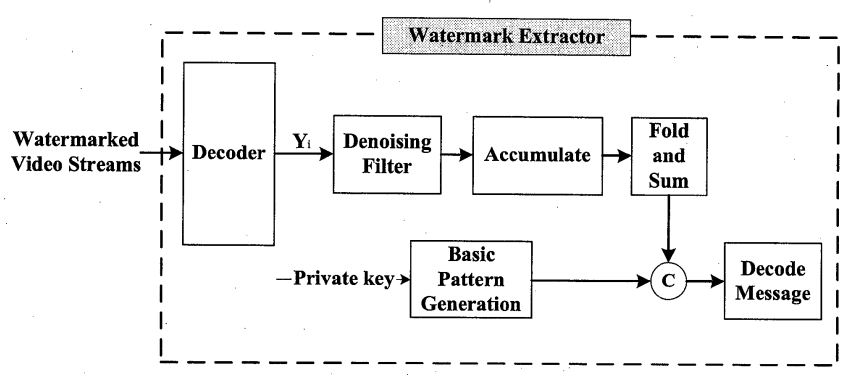

Fig. 3 Block diagram of real-time watermark extraction process.

and unit variance. Then, messages are encoded into each random pattern and 2-D watermark patterns are generated using these encoded patterns. To resist resizing attack, the size of the 2-D patterns should be smaller than the size of attacked videos by resizing attack. Next, the 2-D patterns are enlarged according to the size of host video and tiled. By enlarging the 2-D patterns, the 2-D patterns become lowfrequency patterns, survive video distortions, but have high possibility to be visible. Thus, we need additional imperceptible technique, called dithering, for reducing visible artifacts, that is discussed in Sect.3.3. Before directly embedding the watermarks into frames, we should consider local scaling factor by using HVS function to improve invisibility and robustness. We discuss local scaling in detail in Sect. 3.2. Finally, the watermarked frames $Y_{i}$ are obtained by adding the dithered watermarks to host video frames $X_{i}$ considering local scaling factor. For boosting detector performance, the same watermark is inserted into a fixed number of $t$ seconds (i.e., the same watermark is embedded for a host video at $30 \mathrm{fps}$ during $t \times 30$ consecutive frames).

\subsubsection{Watermark Extraction}

Figure 3 depicts the watermark extraction scheme. We use a blind watermark detector performed by normalized cross correlation. The extraction process is the inverse of the embedding process.

Let $Y_{i}$ be a set of watermarked video frames $(1 \leq i \leq$ $N)$. First, $M$ random patterns are created by using the same private key as, watermark embedding. In aspect of host signals, the watermark is regarded as noise. Therefore, each frame $Y_{i}$ is sent to a denoising filter and we obtain the estimated watermark patterns by subtracting the filtered video 
frame from $Y_{i}$. Adaptive Wiener filter [19] is employed as our denosing filter and a $3 \times 3$ window is used to compute mean and variance values of an individual pixel. The estimated watermark pattern is given by

$$
W^{\prime}(i, j)=\frac{V_{W^{\prime}}(i, j)}{V_{W^{\prime}}(i, j)+V_{Y}(i, j)}\left[Y(i, j)-M_{Y}(i, j)\right]
$$

where $V(i, j)$ and $M(i, j)$ are the local mean and variance value for the $(i, j)$ pixel location. Since detector has no knowledge of probability distribution and properties of watermark pattern, we can replace $V_{W^{\prime}}(i, j)$ with the mean value of $V_{Y}$.

In order to enhance the estimated watermark energy, we accumulate the estimated watermark from the correspondence $Y_{i}$ during $t$ seconds. After accumulation during $t \mathrm{sec}-$ onds, the watermark is constructed by folding and summing it simultaneously. If normalized cross correlation value $C$ exceeds a preset threshold, the hidden messages are correctly extracted. Experimentally, we determine the preset threshold depending on error probability model. We will talk the analysis of error probability in Sect. 3.4.

\subsection{Real-Time Issue - HVS}

\subsubsection{Reducing Computing Time}

As mentioned above, there are specific media processing approaches such as general purpose processors (GPP) with SIMD execution, vector processors, FPGA, ASIC, and VLIW. We are motivated by SIMD execution for GPPs owing to following reasons [20], [21].

- SIMD is to define general purpose instructions that can benefit a large number of applications in different domains of multimedia and communications.

- SIMD is to substantially improve performance for compute-intensive applications.

- Time consuming code has characteristic that localized and recurring computations are performed on the data.

In SIMD approach, our basic idea is to use sub-word parallelism, where a 64-bit register is treated with 8 8-bit values, because the large majority of information can be stored in 8-bit data. We can reduce computing time by partitioning a 64-bit operation to handle multiple narrow operations in parallel. Figure 4 shows an example of SIMD operation for sum of partial product. First, a 64-bit register is partitioned 4 16-bit operations. Then, 4 32-bit calculations result from multiplying 2 16-bit operations in parallel. Finally, we get product result by adding 232 -bit operations in parallel. Generally, we need $64 \times 64$ multiplications without SIMD operation, while $4 \times 16 \times 16$ multiplications plus miscellaneous additions with SIMD operation. If GPPs apply SIMD approach, the cost of the system is significantly reduced. Therefore, we can speed up computing time up to eight times faster with Intel's multimedia extension (MMX) technology.

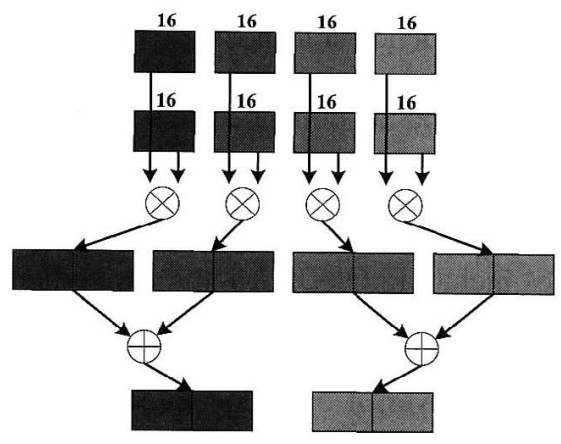

Fig. 4 An example of SIMD operation for sum of partial product.

\subsubsection{Simplification of HVS Function}

A HVS function plays an important role in watermarking system, especially robust and invisible watermarking. A NVF is commonly used as a HVS function. According to the properties of NVF, watermark strength is stronger in edge or textured regions than that in flat regions. This function includes time consuming routine over the rest of other watermark embedding steps, because it computes local mean, local variance, and division operations as shown in Eq. (2).

$$
N V F(i, j)=\frac{1}{1+\theta \sigma^{2}(i, j)}, \text { where } \theta=\frac{D}{\sigma_{\text {max }}^{2}}
$$

The $\sigma^{2}(i, j)$ is a local variance, $\sigma_{\max }^{2}$ is the maximum local variance, and $D$ is experimentally determined $[50,100]$. However, since NVF function results in floating point values between 0 and 1 , it cannot be directly implemented by MMX technology.

Therefore, we employ an edge detector while a NVF concept is preserved and utilize separable property for adaptation of MMX. We focus on a 2-D linear separable filter with a $3 \times 3$ rectangular kernel of constant coefficients. As shown in Fig. 5, separable means that 2-D linear filter can be decomposed into a one dimensional horizontal filter and a one dimensional vertical filter. The separable $M \times N$ filter is more computationally efficient than a non-separable one, because it requires only $M+N$ multiplications instead of $M \times N$ multiplications. In our watermarking, eight compass operators are adopted as an edge detector (see Fig. 5 (c)). The compass operators measure gradients in selected number of directions. An anti-clockwise circular shift of the eight boundary elements gives a 45 degree rotation of the gradient direction. As a consequence of this consideration, we adopted the contents-adaptive embedding rule in the NVF method and slightly modified it suitable for practical situation as below.

$$
\Lambda=(C-E S) \cdot \alpha+E S \cdot \beta
$$

where $C$ is a constant value that limits the upper bound of ES energies, $\alpha$ is a strength parameter for edged or textured areas and $\beta$ is a strength parameter for flat areas. ES represents strength values from our compass operators. Using 


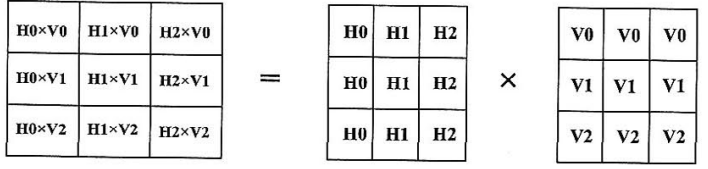

(a) Property of 2-D separable linear filter

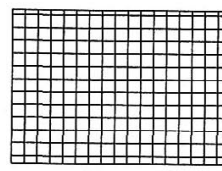

Frame

(b) Filter operation

\begin{tabular}{|r|r|r|}
\hline 1 & 1 & 1 \\
\hline 0 & 0 & 0 \\
\hline-1 & -1 & -1 \\
\hline \multicolumn{4}{|c}{} \\
N
\end{tabular}

\begin{tabular}{|c|c|c|}
\hline 1 & 1 & 0 \\
\hline 1 & 0 & -1 \\
\hline 0 & -1 & -1 \\
\hline \multicolumn{3}{|c|}{ NW }
\end{tabular}

\begin{tabular}{|c|c|c|}
\hline-1 & -1 & -1 \\
\hline 0 & 0 & 0 \\
\hline 1 & 1 & 1 \\
\hline
\end{tabular}

\begin{tabular}{|c|c|c|}
\hline-1 & -1 & 0 \\
\hline-1 & 0 & 1 \\
\hline 0 & 1 & 1 \\
\hline
\end{tabular}

SE
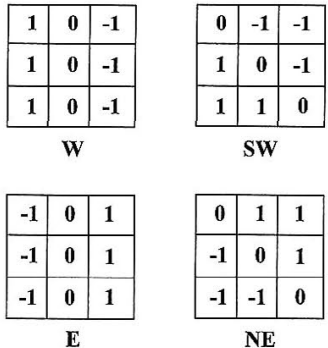

(c) Example of 2-D separable linear filter : compass operator

Fig. 5 A 2-D separable linear filter.

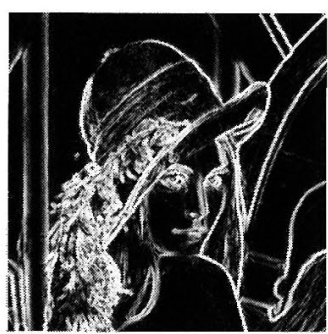

(a)

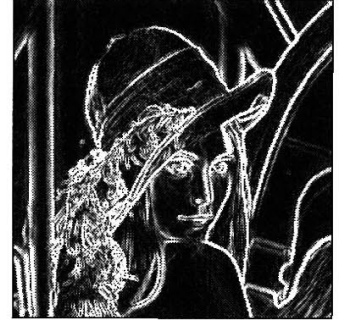

(b)
Fig. 6 (a) NVF-based mask $(D=100)$ and (b) proposed mask for Lenna image. Since the result of NVF function is between 0 and 1, the NVF mask is scaled for viewing purposes.

this HVS function $\Lambda$, the watermark has a strength range between $\alpha$ and $\beta$. Therefore, watermarks could be controlled by adjusting the parameters $\alpha$ and $\beta$ according to a tradeoff between watermark visibility and robustness. The two masks of the examined image are shown in Fig. 6.

\subsection{Perceptual Quality Issues - Dithering}

This section proposes a method to improve the visual quality of low frequency watermark by applying dithering technique. As explained before, we achieve robustness of the watermark by considering low frequency components, because low frequency components are changing little with common image and video processing, especially data compression, low-pass filtering, digital-to-analog (D/A) and analog-to-digital (A/D) [22]. However, the visual noise by low frequency watermark is easily recognized by human eyes and affects the quality of original contents. Dithering

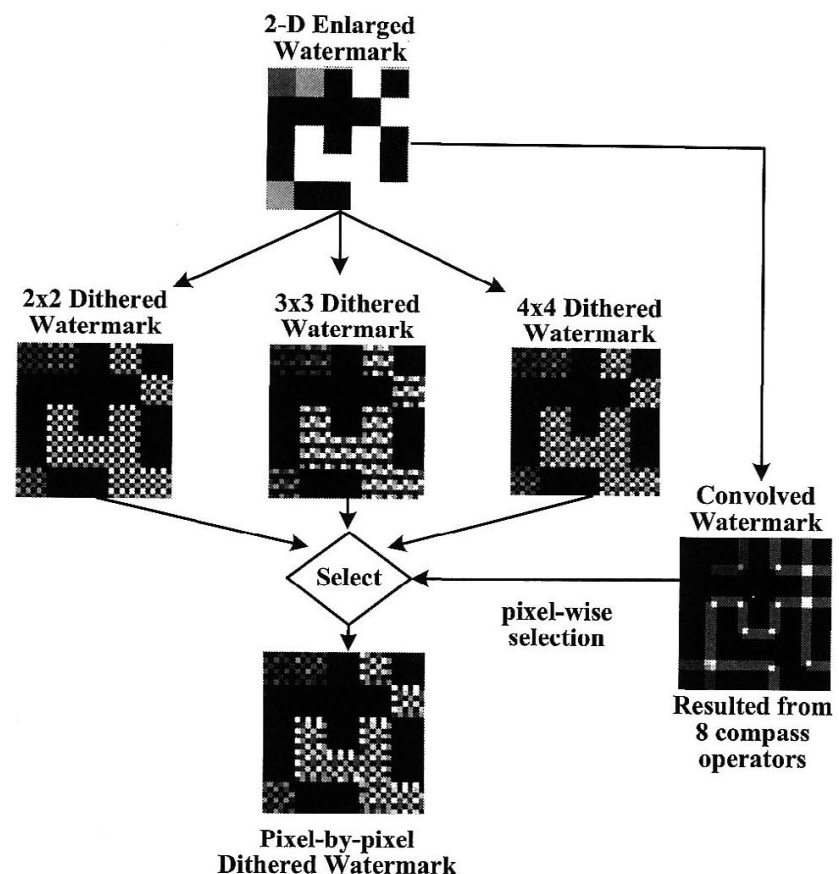

Fig. 7 Overview of pixel-by-pixel variable dithering method.

is used as printing on a 1-bit printer, but we utilize it to reduce the visible artifacts. We design pre-defined matrices that have different sizes such as $2 \times 2,3 \times 3$, and $4 \times 4$. The values of each matrix follow in numerical order and are normalized (e.g., the values in $2 \times 2$ matrix are composed of 0 , $0.333,0.666$, and 1 ). Then, 32 -D dithered watermarks are created by using these matrices. Finally, a value of a smooth region of 2-D enlarged watermark is taken from the dithered value using larger size of matrix, whereas a value of a detail region is taken using smaller size of matrix as shown in Fig. 7. Using the edge strength in Sect. 3.2.2, smooth and detail regions are determined. This step is basically similar to the pixel-by-pixel variable dithering method outlined in [23]. In experiment, we demonstrate improvement of visual quality by utilizing dithering technique.

\subsection{False Positive Probability Analysis}

Since error probability is related to the reliability of the system, it is important to analyze error probability of watermark detection systems. False positive error $\left(f_{p}\right)$ occurs when the detector incorrectly indicates that a watermark is present and false negative error $\left(f_{n}\right)$ occurs when the detector incorrectly indicates the absence of a watermark [24]. The detector must carefully choose a decision threshold depending on the error probability. In watermarking applications such as broadcasting monitoring to confirm that advertisement is aired, false negative error is more serious than false positive error. However, false positive is important in copy control applications to require the detector to extract covert messages. In practice, it is difficult to analyze false negative error due to the wide variety of possible attacks. From this point of view, we use false positive error probability to 
choose a preset threshold at the maximum $f_{p}$ (e.g., $10^{-7}$ ). False positive error probability of the correlation detector follows Gamma distribution model, since we take the maximum value from cross correlation. Gamma distribution is defined as below [14]

$$
f(x ; a, b)= \begin{cases}\frac{1}{b^{a} \Gamma(a)} x^{a-1} e^{-x / b} & \text { if } x \geq 0 \\ 0 & \text { otherwise }\end{cases}
$$

where $x$ is a continuous random variable. Parameters $a$ and $b$ are calculated using the mean and variance of the random variable $x$ as follows:

$$
E(x)=\mu_{x}=a b, \quad V(x)=\sigma_{x}^{2}=a b^{2}
$$

Through experiments, we determined the parameter of Gamma distribution and used to choose the threshold of the watermark detector.

\section{Simulation Results}

\subsection{Test Environment}

We tested the proposed video watermarking scheme on an Intel Pentium IV CPU with a $3.6 \mathrm{GHz}$ core, $2 \mathrm{~GB}$ DDR2 RAM, and ATI X1600 graphics card. We used 4 MPEG-2 HD host videos at $30 \mathrm{fps}$ which contain more than 20 different scenes and features (see Fig. 8).

To embed watermarks, watermarking embedding variables $M, L$, and $t$ were set as 256, 4200, and [1,5], respectively. Also we adjusted HVS $\Lambda$ value of Eq. (3) to have between 1 and 6 . Since we assumed that covert messages were ASCII code, for example "WATERMARK", we set $M=256$ to represent an 8-bits character. With these setting, a 2 -D watermark pattern is formed $240 \times 210$ dimensions. Then, the 2-D watermark pattern is enlarged with 6 and 4.5 times in width and height, respectively and is tiled. As described before, the enlarged watermark causes blocking artifact, but we reduce this artifact by the dithering technique.

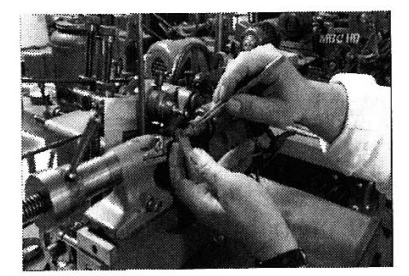

(a)

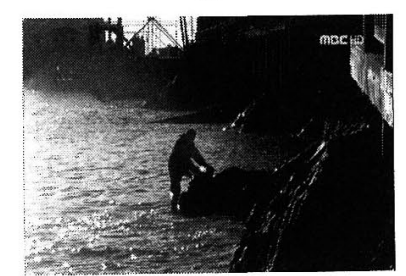

(c)

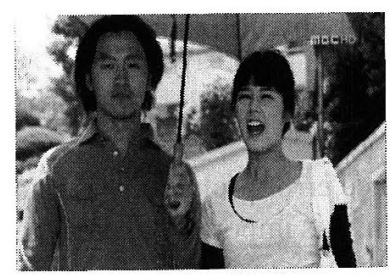

(b)

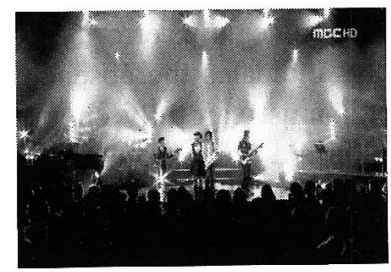

(d)
Fig. 8 Snapshot examples of 4 test videos : (a) Documentary at 24.6 Mbps, (b) Drama at $32.2 \mathrm{Mbps}$, (c) Movie at $40.9 \mathrm{Mbps}$, and (d) Music show at $32.5 \mathrm{Mbps}$. Test sets contain a number of scenes and different features.

\subsection{Real-Time Performance}

Real-time watermarking system should embed watermarks into host videos at $30 \mathrm{fps}$ in $0.03 \mathrm{sec}$. per a frame. That is, three sub-functions for decoding video streams, embedding watermarks, and displaying watermarked video on a screen should be done in $0.03 \mathrm{sec}$. per a frame. We adopted Intel VTune Performance Analyzer that identifies the bottleneck of the system and calculates processing time of each function. Based on the analysis, the HVS function part for invisibility took about $70 \%$ of the total computing time. Also, we compared the processing time with pure C code NVFbased watermarking system. The NVF processing time in pure $C$ system was $0.0255 \mathrm{sec}$. and the total processing time was $0.0590 \mathrm{sec}$., beyond of the time limit per a frame. However, the processing time of our simplified HVS mask method was $0.0039 \mathrm{sec}$. to get local scaling factor and the total processing time was $0.0287 \mathrm{sec}$, still under the processing limit time per a frame. It means that our scheme can decode HD-resolution bitstreams, embed robust watermark with HVS, and display the watermarked frames on a screen within $0.03 \mathrm{sec}$. From the results, we optimized the total processing time from $0.0590 \mathrm{sec}$. to $0.0287 \mathrm{sec}$. and thus achieved about $51 \%$ performance enhancement. Table 1 shows the processing time for watermark embedding. In both systems, the decoding and displaying process took a constant time, 0.013 and $0.005 \mathrm{sec}$. per a frame, respectively.

We observed that the MMX coding considerably reduced the time complexity of a whole embedding processing, especially that of the HVS masking function. However, its instructions operate with only integer data and support 64-bit memory accesses at most. In particular, programming with streaming SIMD extension (SSE) that provides floating-point instructions and memory control instructions and supports up to 128-bit memory accesses can enhance the ability to perform HVS function. Results show that our MMX-based watermarking scheme satisfies real-time requirement.

\subsection{Visual Quality}

Signal processing applications measure peak signal to noise

Table 1 Time complexity comparisons between watermarking system implemented with pure $\mathrm{C}$ code and MMX code. Execution time of each function in Fig. 2 is summarized in detail. Basic pattern generation, scaling

\begin{tabular}{|c|c|c|c|c|c|c|}
\hline & \multirow{2}{*}{$\begin{array}{l}\text { Decode } \\
\text { Time }\end{array}$} & \multicolumn{3}{|c|}{ Watermark Embedding } & \multirow{2}{*}{$\begin{array}{l}\text { Display } \\
\text { Time }\end{array}$} & \multirow{2}{*}{$\begin{array}{l}\text { Total } \\
\text { Time }\end{array}$} \\
\hline & & HVS & $\begin{array}{l}\text { Local } \\
\text { Scaling }\end{array}$ & $\begin{array}{l}\text { ADD, } \\
\text { MUL }\end{array}$ & & \\
\hline $\begin{array}{l}\text { Pure C } \\
\text { System }\end{array}$ & \multirow{2}{*}{0.013} & $\begin{array}{c}0.0255 \\
\text { (NVF }[11])\end{array}$ & 0.0069 & 0.0086 & \multirow{2}{*}{0.005} & 0.0590 \\
\hline $\begin{array}{l}\text { MMX } \\
\text { System }\end{array}$ & & $\begin{array}{c}0.0039 \\
\text { (our HVS) }\end{array}$ & 0.0040 & 0.0028 & & 0.0287 \\
\hline
\end{tabular}
and tiling, and dithering processes were made at pre-process (unit: sec). 
ratio (PSNR) to represent the quantitative quality. The average PSNR values after watermark embedding with the proposed dithering technique was $46.3 \mathrm{~dB}$ for Documentary, $45 \mathrm{~dB}$ for Drama, $43 \mathrm{~dB}$ for Movie, and $44.5 \mathrm{~dB}$ for $\mathrm{Mu}-$ sic show as shown in Fig. 9. The PSNR difference between with dithering and without dithering was around $1.5 \mathrm{~dB}$.

In order to analyze the affection of original video clips after watermark insertion, we performed the similar experiment for fidelity testing [25]. Ten observers who are famil-

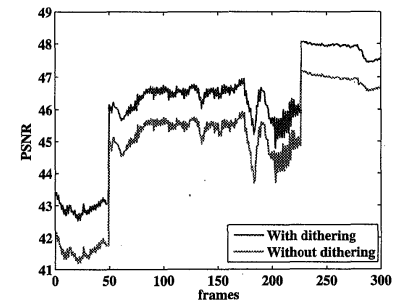

(a)

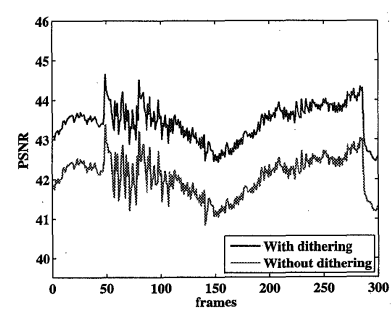

(c)

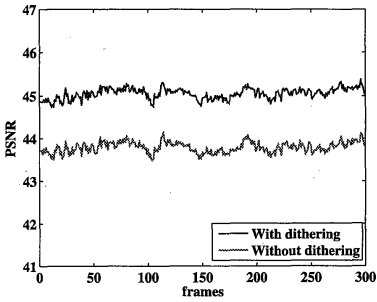

(b)

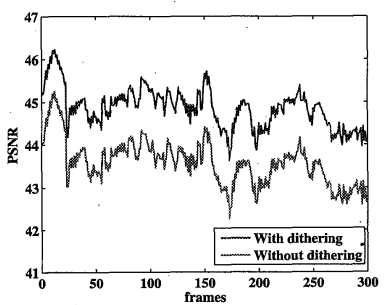

(d)
Fig. 9 PSNR values in first 300 frames after watermark embedding with and without dithering method: (a) Documentary, (b) Drama, (c) Movie, and (d) Music show. iar with the details of the watermark algorithm and are able to detect visual artifacts took part in the examination. The same video clip, once with and once without watermark, is randomly displayed on a HD $(1920 \times 1080$ pixel resolution $)$ size screen in each trial. After four trials, all observers reported that no one was able to determine reliably the presence of the watermark in any case. In addition, we subjectively evaluated the quality of the watermarked videos using the ITU-R Rec. 500 quality rating scale [24]. The average score for all trials was better than 4, "Perceptible, but not annoying". Results show that our watermarking scheme can produce high fidelity watermarked videos and have no obvious processing artifacts.

\subsection{Robustness}

All test videos were watermarked using the proposed method. On average, our watermarking process increased the size of the original bitstream by $5.1 \%$. We focused the robustness of our watermarking system against combined attacks commonly happened to high quality videos under practical situations. Watermarked HD MPEG-2 videos, which are between at $25.8 \mathrm{Mbps}$ and at $42.9 \mathrm{Mbps}$, were processed in several ways such as arbitrary-ratio downscaling, frame rate change, and transcoding to MPEG-4 formats that are between at $6.7 \mathrm{Mbps}$ and at $0.4 \mathrm{Mbps}$. People scale down HD contents to VGA or QVGA contents for playing on portable devices and convert MPEG-2 formats to MPEG4 formats to reduce file sizes for easy manipulations or network transference. Figures 10, 11, and 12 show experimental results against these combined attacks. The $x$ axis repre-

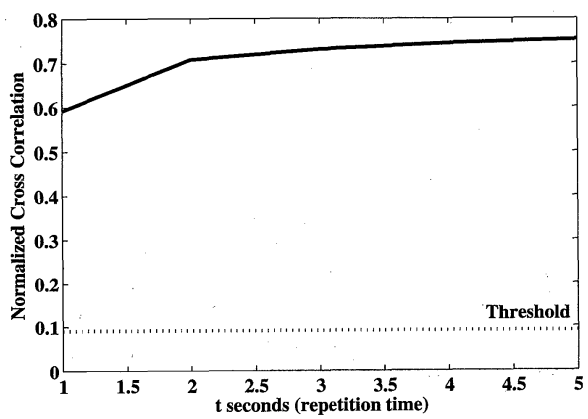

(b) Drama at $34.1 \mathrm{Mbps}$

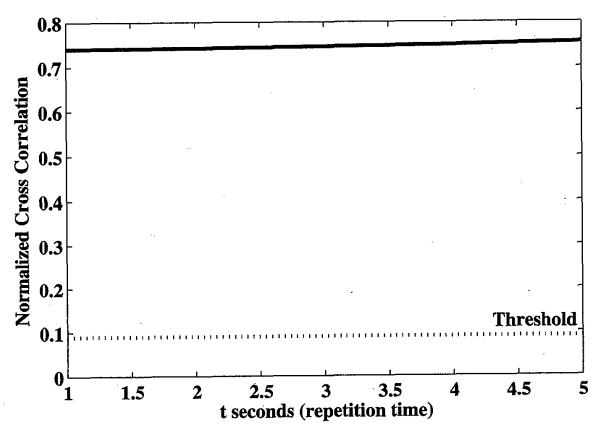

(c) Movie at $42.9 \mathrm{Mbps}$

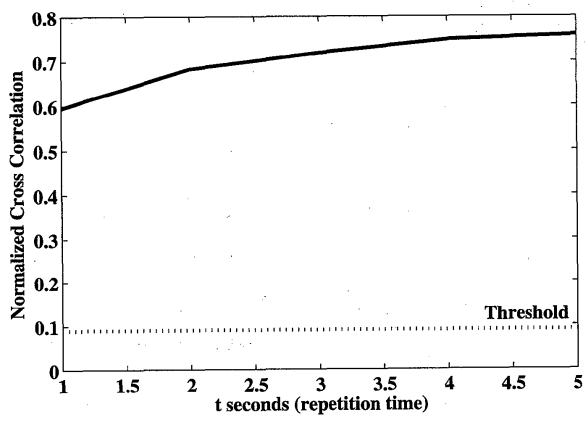

(d) Music show at 34.1 Mbps

Fig. 10 No attack. 


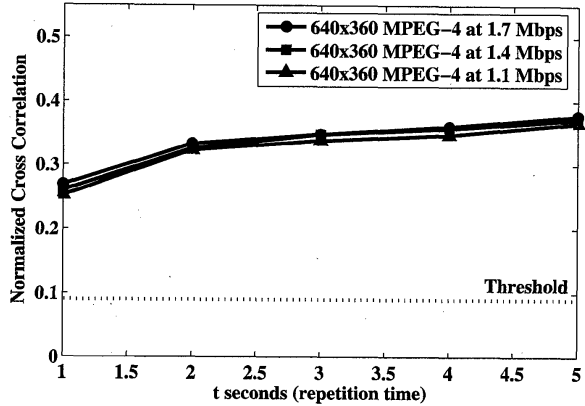

(a) Documentary

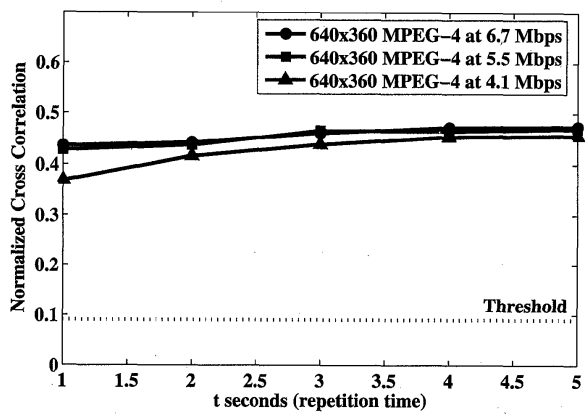

(c) Movie

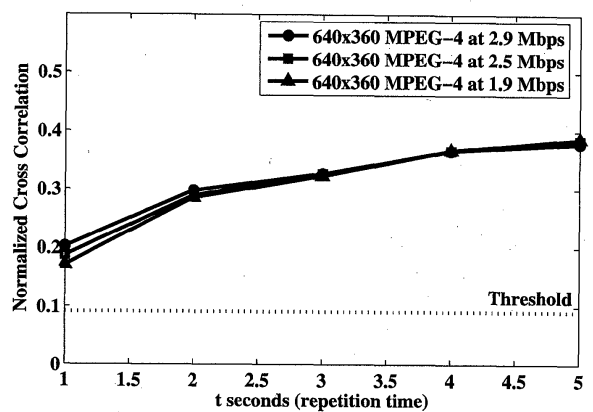

(b) Drama

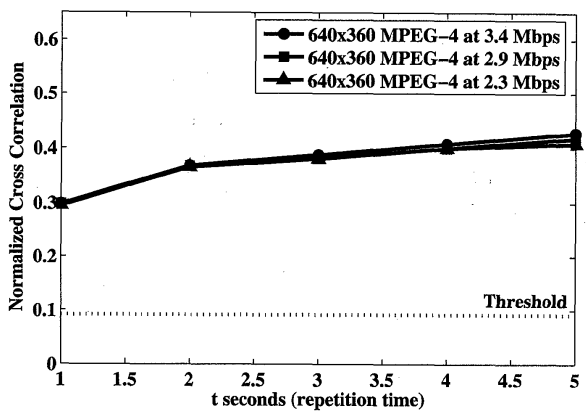

(d) Music show

Fig. 11 Combined attack: downscale to $640 \times 360$, convert into MPEG-4 format, and change bit rates.

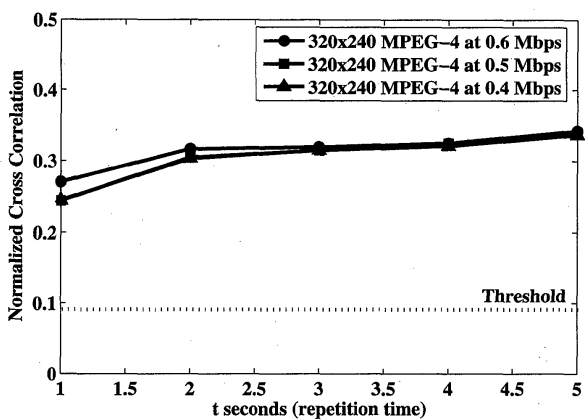

(a) Documentary

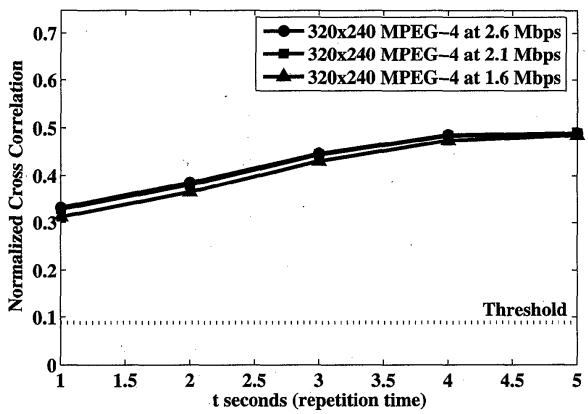

(c) Movie

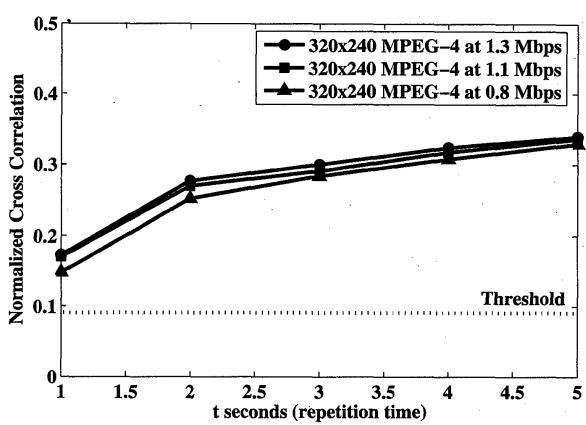

(b) Drama

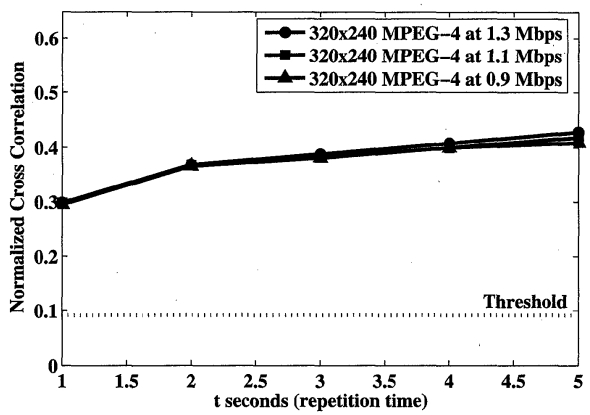

(d) Music show

Fig. 12 Combined attack: downscale to $320 \times 240$, convert into MPEG-4 format, and change bit rates.

sents the repetition number of the same watermark and the $y$ axis represents the normalized correlation values in that repetition time. We determine the preset threshold $(T=0.09)$ to have $f_{p}=10^{-7}$. This allows us to be confident that the specified error rate will not be exceeded.
Our watermarks are more strongly embedded in edge and textured areas (i.e., middle and high frequency components). Therefore, the watermark correlation values in videos at higher bit-rates are higher than those in videos at lower bit-rates because the bitstream at low bit-rates car- 
Table 2 Summarization of the robustness against various attacks in the proposed real-time watermarking system (threshold $=0.09$ ).

\begin{tabular}{lcccc}
\hline & Documentary & Drama & Movie & Music show \\
\hline Original $(1920 \times 1080)$ & 0.64 & 0.59 & 0.74 & 0.59 \\
Resize to SXGA $(1280 \times 1024)$ & 0.52 & 0.47 & 0.70 & 0.54 \\
Resize to DVD $(720 \times 480)$ & 0.40 & 0.36 & 0.59 & 0.41 \\
Resize to CIF $(352 \times 288)$ & 0.36 & 0.32 & 0.41 & 0.33 \\
Cropping & 0.54 & 0.48 & 0.64 & 0.55 \\
Gaussian Filtering & 0.42 & 0.48 & 0.54 & 0.43 \\
Noise Addition & 0.50 & 0.49 & 0.62 & 0.47 \\
MPEG-1 Conversion & 0.47 & 0.51 & 0.58 & 0.46 \\
\hline
\end{tabular}

ries only visually significant features (low frequency components) of the video. However, all correlation values in videos at low bit-rates largely exceed the threshold for all test videos. This means that our watermarks are correctly extracted despite of severe combined attacks including huge downsizing attack, transcoding attack, and frame rate change attack. Also, we can see that the correlation values become high with the increase of the repetition time.

We applied various image and video processing attacks such as scaling, cropping, Gaussian filtering, noise addition, and MPEG-1 conversion and summarized the results in the Table 2. As we expected, the average normalized correlation values were larger than the threshold, 0.09 , satisfying false positive error probability of $10^{-7}$. It means that the proposed watermarking system is robust against these attacks.

Through experiments, we selected attacks by focusing on video manipulations which could happened frequently to HD videos and did not take into account common signal processing or geometric attacks in image watermarking. However, we applied robust watermarking techniques such as spread-spectrum method and human visual masking method that are already proved in other researches, thus we expect that our watermarks would be robust against those kinds of attacks.

\section{Conclusions}

As digital content markets and infrastructures are emerging, high quality contents are becoming the center of market shares. Digital watermark techniques should come up with market currencies. We presented practical, real-time, and robust video watermarking scheme in spatial domain with the focus on real-time performance and robustness against downscaling resolution, frame rate change, and transcoding. Our contribution is that we suggested a simplified HVS method to decrease processing time and increase multimedia manipulation efficiency and talked about system implementation using the MMX technology. Since our HVS method provides low time complexities and high performance capabilities, it is well-suited for real-time watermarking applications. We also proposed a dithering technique based on HVS to enhance visual quality of watermarked HD videos. We implemented the watermarking system that includes the simplified HVS function and the variable dither- ing method and showed the best performances in both realtime processing and robustness. All these methods could be applied to other video watermarking schemes and other video processing applications. Since our scheme is suitable for directshow built-in applications, one practical use is to implement watermarking embedder and detector as directshow filters. Then, our watermarking filter can be connected to any applications for copyright protection.

\section{Acknowledgments}

This work was in part supported by the KOSEF grant NRL program funded by the Korea government(MOST) (No. R0A-2007-000-20023-0), and the IT R\&D program of MIC/IITA(2007-S017-01, Development of user-centric contents protection and distribution technology)

\section{References}

[1] H.T. Sencar, M. Ramkumar, and A.N. Akansu, Data Hiding Fundamentals and Applications, Elsevier Academic Press, UK, 2004.

[2] W. Zeng, H. Yu, and C.Y. Lin, Multimedia Security Technologies for Digital Rights Management, Elsevier Academic Press, USA, 2006.

[3] C. Busch, W. Funk, and S. Wolthusen, "Digital watermarking: From concepts to real-time video applications," IEEE Comput. Graph. Appl., vol.19, no.1, pp.25-35, 1999.

[4] G.C. Langelaar and R.L. Lagendijk, "Optimal differential energy watermarking of DCT encoded images and video," IEEE Trans. Image Process., vol.10, no.1, pp.148-158, 2001.

[5] G.C. Langelaar, R.L. Lagendijk, and J. Biemond, "Real-time labeling of MPEG-2 compressed video," J. Visual Commun. Image Represent, vol.9, no.4, pp.256-270, 1998.

[6] H. Ling, Z. Lu, and F. Zou, "New real-time watermarking algorithm for compressed video in VLC domain," Int. Conf. Image Processing, vol.4, pp.2171-2174, 2004.

[7] Y. Wang and A. Pearmain, "Blind MPEG-2 video watermarking robust against geometric attacks: A set of approaches in DCT domain," IEEE Trans. Image Process., vol.15, no.6, pp.1536-1543, 2006.

[8] I.J. Cox, J. Kilan, T. Leighton, and T. Shamoon, "Secure spread spectrum watermarking for multimedia," IEEE Trans. Image Process., vol.6, no.12, pp.1673-1687, 1997.

[9] J.F. Delaigle, C.D. Vleeschouwer, and B. Macq, "Watermarking algorithm based on a human visual model," Signal Process., vol.66, no.3, pp.319-335, 1998.

[10] T. Xianghong, X. Shuqin, and L. Qiliang, "Watermarking for the digital images based on model of human perception," IEEE Int. Conf. Neural Networks and Signal Processing, pp.1509-1512, 2003.

[11] S. Voloshynovskiy, A. Herrigel, N. Baumgartner, and T. Pun, 
"A stochastic approach to content adaptive digital image watermarking," Int. Workshop on Information Hiding, vol.1768, pp.212-236, 1999.

[12] M. Kutter, F. Jordan, and F. Bossen, "Digital signature of color images using amplitude modulation," Storage Retrieval for Image Video Databased V, Proc. SPIE Electronic Imaging, pp.518-526, 1997.

[13] C.H. Lee and H.K. Lee, "Improved autocorrelation function based watermarking with side information," J. Electron. Image, vol.14, no.1, pp.1-13, 2005.

[14] H.Y. Lee, H.S. Kim, and H.K. Lee, "Robust image watermarking using invariant features," Opt. Eng., vol.45, no.3, pp.1-11, 2006.

[15] G. Petitjean, J.L. Dugelay, S. Gabriele, C. Rey, and J. Nicolai, "Towards real-time video watermarking for system-on-chip," Proc. IEEE Int. Conf. on Multimedia and Expo, pp.597-600, 2002.

[16] N.J. Mathai, A. Sheikholeslami, and D. Kundur, "VLSI implementation of a real-time video watermark embedder and detector," Proc. IEEE Int. Symposium on Circuits and Systems, pp.772-775, 2003.

[17] I. Echizen, T. Yamada, Y. Fujii, S. Tezuka, and H. Yoshiura, "Realtime video watermark cmbedding system using software on personal computer," Proc. IEEE Int. Conf. on System, Man and Cybernetics, pp.3369-3373, 2005.

[18] I. Echizen, K. Tanimoto, T. Yamada, M. Dainaka, S. Tezuka, and H. Yoshiura, "PC-based real-time video watermark embedding system with standara video interface," Proc. IEEE Int. Conf. on System, Man and Cybernetics, pp.267-272, 2006.

[19] I.G. Karybali and K. Berberidis, "Efficient spatial image watermarking via new perceptual masking and blind detection schemes," IEEE Trans. Inf. Forensics and Security, vol.1, no.2, pp.256-274, 2006.

[20] Intel architecture MMX technology developer's manual, Intel Corporation, 1996.

[21] D. Bistry, The complete guide to MMX technology, McGraw-Hill, 1997.

[22] J. Huang, Y.Q. Shi, and Y. Shi, "Embedding image watermarks in DC components," IEEE Trans. Circuits Syst. Video Technol., vol.10, no.6, pp.974-979, 2000.

[23] H.Z. Hel-Or, X.M. Zhang, and B.A. Wandell, "Adaptive cluster dot dithering," J. Electron. Imaging, vol.8, no.2, pp.133-144, 1999.

[24] I.J. Cox, M.L. Miller, and J.A. Bloom, Digital Watermark, Morgan Kaufmann Publishers, USA, 2002.

[25] J. Lubin, J.A. Bloom, and H. Cheng, "Robust, content-dependent, high-fidelity watermark for tracking in digital cinema," Security and Watermarking of Multimedia Contents V, Proc. SPIE Electronic Imaging, pp.536-545, 2003.

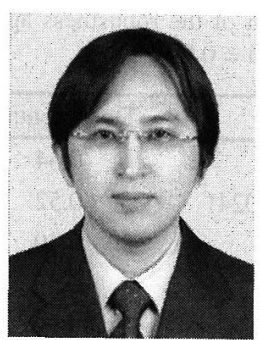

Hae-Yeoun Lee received his M.S. and Ph.D. degrees in computer science from Korea Advanced Institute of Science and Technology, Korea, in 1997 and 2006 respectively. From 2001 to 2006, he was with Satrec initiative, Korea. Currently, he is a professor in Kumoh National Institute of Technology, Republic of Korea. His major interests are digital watermarking, image processing, remote sensing and digital rights management.

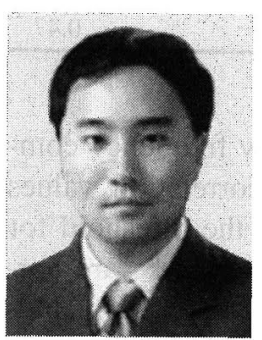

Dong-Hyuck Im received a B.S. degree in computer science from Yonsei University, Korea, in 2001 and a M.S. degree in computer science from Korea Advanced Institute of Science and Technology, in 2006. He is currently pursuing a $\mathrm{Ph} . \mathrm{D}$. degree in computer science at KAIST. His research interests are digital watermarking, digital fingerprinting, and digital forensic.

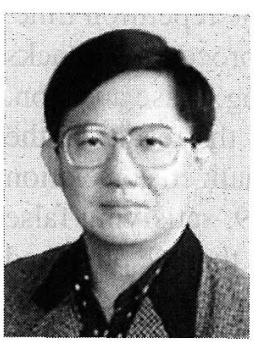

Heung-Kyu Lee received a B.S. degree in electronics engineering from Seoul National University, Seoul, Korea, in 1978, and M.S. and Ph.D. degrees in computer science from Korea Advanced Institute of Science and Technology, Korea, in 1981 and 1984, respectively. Since 1986 he has been a professor in the Department of Computer Science, KAIST. His major interests are digital watermarking, digital fingerprinting, and digital rights management.

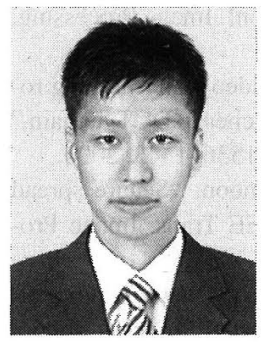

Kyung-Su Kim received the B.S. degree in Computer Engineering from Inha University, Republic of Korea, in 2005, and the M.S. degree in Computer Science from Korea Advanced Institute of Science and Technology (KAIST), Republic of Korea, in 2007. He is currently working toward his Ph.D. degree in Multimedia Computing Lab., Dept. of EECS, KAIST. His research interests include image/video watermarking and fingerprinting, error concealment method, information security, multimedia signal processing, and multimedia communications. 